\title{
The beta coefficient for the number of factors in the minres factor analysis
}

\author{
SHIGEO KASHIWAGI ${ }^{\mathbf{1}}$ \\ Department of Psychology, Faculy of Humanities and Social Sciences, Chiba University, Chiba 260
}

\begin{abstract}
A new criterion to estimate the number of factors in multiple factor analysis was proposed, based on the mathematical relationships between the principle of the linear regression analysis passing through the origin and that of the minres factor analysis by Harman (1976). The criterion is the beta coefficient for the linear regression line defined by the elements in each of the original and the reproduced correlation matrices, and it is obtained with the increase of the number of factors. Numerical examples are presented and some supplemental issues are discussed.
\end{abstract}

Key words: coefficient of linear regression line, principal component analysis, minres factor analysis, number of factors, Guttman's algebraic lower bounds, correlational coefficient.

It may be said that there exist two kinds of the criteria concerning the number of factors in multiple factor analysis. The former kind of the criteria are proposed on the basis of the notion of the distribution of eigenvalues such as Guttman's (1954) weaker and stronger lower bounds, and the latter kind of them on the basis of the notion of the statistical test of likelihood ratio (Lawley \& Maxwell, 1971; Jöreskog, 1962). The AIC criterion which was discussed by Akaike (1971) and which was advocated to use for the estimation of the number of factors may be classified into the latter kind of the criteria.

Although the alpha factor analysis (Kaiser \& Caffrey, 1965) and the image factor analysis (Guttman, 1953) might be said to be proposed in relation to the weaker and stronger lower bounds of Guttman, respectively, the both types of the factor analyses do not seem to discuss so often at the present time.

1 The author owes Mrs. Yoshimi Tanaka of the Japan Psychological Corporation (Nihon Seishin Gijutsu Kenkyusho) for the computation of this time, and would like to extend his sincere thanks to her efforts.
On the contrary, the factor analytic model based on the principle of maximum likelihood, which may give the statistical bases for the latter kind of the criteria, have still discussed very frequently by many statisticians. However, from the applied psychological point of view, the both types of the factor analytic models and their related criteria for the number of factors may not always give satisfactory answers. In particular, with regard to the number of factors brought about through the both kinds of the criteria, the former kind of them tend to give the numbers of factors which may be determined dependently upon the number of the variables or size in a correlation matrix while the latter kind of them tend to give the numbers of factors which may be determined dependently upon the number of samples. Thus, even assuming that a result through the maximum likelihood method could be properly obtainable, the determination of the appropriate number of samples may be an important matter in using the latter kind of the criteria.

Although the choice concerning with which kind of the criterion should be adopted may be done by the user's pur- 
pose of factor analysis, it may be that the former kind of the criteria are preferable to the latter in the sense of their being useful from the practical point of view. Here it should be noted that the usefulness of herein means the assurance of the factorial invariance in the Thurstone's (1947) sense. Of course, even if any one of the algebraic criteria is used, to assure perfectly the factorial invariance may always be impossible in performing factor analysis. However, through the efforts of the improvements of the criteria based on the relationships between the distribution of eigenvalues and the number of factors, a new kind of the criteria can be proposed and they may give much better indicators for the number of factors.

Kaiser and Caffrey (1965) advocated to use the weaker lower bound of Guttman's algebraic criteria which takes care of the eigenvectors corresponding to the eigenvalues which are not smaller than unity. He placed the emphasis on the fact that the criterion gave the good agreements to the empirical results concerning the number of factors which were obtained subjectively, and he asserted that, at the present stage, it is mostly recommendable for the practical use. It seems certain that the criterion is very practical when the number of the variables in a correlation matrix is medium instead of its being extremely small. However, as the eigenvectors corresponding to the eigenvalues which are not smaller than unity are considered based on the criterion, the number of the eigenvectors tends to increase as the number of the variables of a correlation matrix increases. This fact has already been indicated by factor analysts. A way around this kind of the tendency or difficulty will herein be proposed by reconsidering the principle of the minres factor analysis by Harman (1976). The mathematical relationships between the principle of the factor analytic model and that of the linear regression will be investigated before explaining our proposal.

The minres factor analysis aims to minimize the sum of the squared residual errors under a given number of factors. The value of the sum will be greater as the number of the variables in a correlation matrix increases, and it is to be dependent upon the size of the matrix. In order to avoid the dependency, Harman (1976) proposed to use a corrected criterion divided by all the number of the pairs of the variables in a correlation matrix. By the proposal, the possibility of evaluating independently of the size of a correlation matrix the discrepancy between the original correlation matrix and the reproduced one under a given number of factors was investigated. In this paper, other kind of the possibility will be discussed by emphasizing that a way of evaluating independently of the number of the variables the similarity between the original correlation matrix and the reproduced one under a given number of factors should be necessary particularly from the practical point of view. The concept of the similarity of here is very simple and concise, and it will be described by the coefficient beta for the linear regression line passing through the origin based on the principle of the least squares of the sum of the orthogonalized errors. That is the original idea and the explanation for the use of the supplementary concept such as the coefficient of the correlation between the original correlation matrix and the reproduced one will also be added in the last part of this paper.

The Principal Component Analysis and the Linear Regression Estimation

When $R(\mathcal{N} \times \mathcal{N})$ and $A(\mathcal{N} \times m)$ denote a correlation matrix and its principal component matrix, respectively, let $r_{i j}$ and $a_{i k}$ (or $a_{j k}$ ) be an element in each of the matrices $R$ and $A$. Here note that 
$m(\leq \mathcal{N})$ shows a given number of the components. When the following

$$
\phi=\sum_{i}^{N} \sum_{j}^{N}\left(r_{i j}-\sum_{k}^{m} a_{i k} a_{j k}\right)^{2}
$$

is to be minimized with regard to the matrix $A$ under the constraint for the orthogonalization

$$
A^{\prime} A=\lambda_{m},
$$

the principal component matrix $A$ can be obtained as is known very well. Here, $\lambda_{m}$ shows the diagonal matrix each element of which is to be an eigenvalue. And when $\sum_{k}^{m} a_{i k} a_{j k}$ in (1) is shown as $\hat{r}_{i j}$, the relation $r_{i j}=\hat{r}_{i j}+e_{i j}$ can be derived where $e_{i j}$ denotes an element of the residual correlation matrix $R-A A^{\prime}$. Here note that the orthogonal relation $(R-$ $\left.A A^{\prime}\right)^{\prime}\left(R-A A^{\prime}\right)=\lambda_{N-m}$ is to be satisfied. In other words, $e_{i j}$ denotes an element of the orthogonalized error matrix $R-A A^{\prime}$.

The minimization problem (1) under (2) can of course be expressed in terms of the linear regression analysis in the single plane as follows. When the function

$$
\phi=\sum_{i}^{N} \sum_{j}^{N}\left(r_{i j}-\hat{r}_{i j}\right)^{2}=\sum_{i}^{N} \sum_{j}^{N} e_{i j}^{2}
$$

is to be minimized with regard to the matrix $A$ under the constraint

$$
\left(R-A A^{\prime}\right)^{\prime} A=0_{N m},
$$

$\hat{r}_{i j}$ can be estimated. Then, when $r_{i j}$ and $\hat{r}_{i j}$ are taken as the predicted (or criterion) variable and the criterion (or predicted) one, respectively, the least squares estimate for the coefficient of the linear regression line passing through the origin is to be unity from the theoretical point of view when the number of the components $m$ is true. That is to say, the angle between the principal axis, which is to be determined by $r_{i j}$ and $\hat{r}_{i j}$, and the abscissa in the regression plane should be $\pi / 4$. Here, the matrix $0_{N m}$ in the formula (4) shows a zero matrix.

\section{The Minres Factor Analysis and the Linear Regression Estimation}

Let the correlation matrix the elements of the diagonal of which are all zeroes be $R_{0} \quad(=R-I) \quad(\mathcal{N} \times \mathcal{N})$. When the communalities $H^{2}$ are inserted into the diagonal of $R_{0}$, we can show as $R^{*}=$ $R_{0}+H^{2}$ where $H^{2}$ is to be a diagonal matrix. $h_{j}^{2}$ and $r_{i j}{ }^{*}$ will be shown as an element of $H^{2}$ and an off-diagonal one of $R^{*}$, respectively. According to Harman (1976), the minres factor analysis assumes that the estimated values of communalities $h_{i}{ }^{2} \mathrm{~s}$ should all be equal to the corresponding calculated values of communalities $\hat{h}_{i}{ }^{2} \mathrm{~s}$, where $\hat{h}_{i}^{2}=\sum_{k}^{m} a_{i k}{ }^{2}$. When the function satisfying the assumption concerning the estimation of the communalities

$$
\begin{aligned}
\phi^{*} & =\sum_{i}^{N}\left(h_{i}{ }^{2}-\hat{h}_{i}{ }^{2}\right)^{2}+\sum_{i \neq}^{N} \sum_{j}^{N}\left(r_{i j}{ }^{*}-\sum_{k}^{m} a_{i k}{ }^{*} a_{j k}{ }^{*}\right)^{2} \\
& =\sum_{i \neq}^{N} \sum_{j}^{N}\left(r_{i j}{ }^{*}-\hat{r}_{i j}\right)^{2}=\sum_{i \neq}^{N} \sum_{j}^{N} e_{i j}{ }^{* 2}
\end{aligned}
$$

is to be minimized with regard to the matrix $A^{*}$ under the constraint

$$
\begin{aligned}
A^{* \prime} A^{*} & =\lambda_{m}{ }^{*} \text { or }\left(R^{*}-A^{*} A^{* \prime}\right)^{\prime} A^{*} \\
& =0_{N \pi}{ }^{*},
\end{aligned}
$$

the minres factor loading matrix $A^{*}$ can be obtained. Here, $a_{i k}{ }^{*}$ (or $a_{j k^{*}}$ ) and $e_{i j}{ }^{*}$ denote an element of $A^{*}$ and the one of $R^{*}-A^{*} A^{* \prime}$, respectively. And, $\hat{r}_{i j} *, \lambda_{m} *$, and, $0_{N m} *$ denote $\sum_{k}^{m} a_{i k} * a_{j k} *$, a diagonal matrix of the eigenvalues, and, a zero matrix, respectively.

By the assumption $h_{i}{ }^{2}=\hat{h}_{i}{ }^{2}$, the formula (5) can be changed into the following function (7) with regard to the upper (or lower) triangle matrix which may or may not include the diagonal elements. That is, when the function 


$$
\begin{aligned}
\phi & =\sum_{i}^{N}\left(h_{i}^{2}-\hat{h}_{i}^{2}\right)^{2}+\sum_{i<}^{N-1} \sum_{j}^{N}\left(r_{i j}{ }^{*}-\hat{r}_{i j} *\right)^{2} \\
& =\sum_{i<}^{N-1} \sum_{j}^{N}\left(r_{i j}{ }^{*}-\hat{r}_{i j}\right)^{2}=\sum_{i<}^{N-1} \sum_{j}^{N} e_{i j}{ }^{2}
\end{aligned}
$$

is to be minimized with regard to $A^{*}$ under the constraint of (6) (Harman, 1976), the matrix $A^{*}$ can be obtained. By changing (5) under the constraint of (6) into (7) under the same constraint, the following may result. That is to say, when $r_{i j}{ }^{*}$ and $\hat{r}_{i j}{ }^{*}$ are taken as the predicted (or criterion) variable and the criterion (or predicted) one, respectively, the estimate by the principle of least squares for the coefficient of the linear regression line passing through the origin is to be unity. The minres factor analysis can thus be expressed as the linear regression analysis passing through the origin in terms of all the elements or of the off-diagonal ones of the upper (or lower) triangle matrix in each of an original and the reproduced correlation matrices under an assumed number of factors. Here note that the term of the number of factors will hereafter used only in discussing the estimation of the true one in multiple factor analysis. Remember then that we used and will use the term of the number of components in discussing the principal component analysis. The mathematical relations above can be derived from the fact that the minres factor analysis assumes to be same in all the values for both estimated and the calculated communalities, and it should be noted that the principal axes method without assuming to be same in both the estimated and the calculated communalities may not be expressed in a simpler form as the minres factor analysis does.

The Estimation of the Number of Factors by the Principle of Linear Regression Analysis

As was discussed at the end of the first part of this paper, the degree of the similarity between a correlation matrix and the reproduced one will herein be described in terms of all the elements or of the offdiagonal elements in the upper (or lower) triangle matrices in the both correlation matrices.

Let $r^{*}$ be a column vector all the elements of which are composed of the corresponding ones in the upper (or lower) triangle matrix including or excluding the elements of the diagonal in a given correlation matrix, and let $\hat{\boldsymbol{r}}^{*}$ be a column vector all the elements of which are composed of the corresponding ones in the upper (or lower) triangle matrix including or excluding the elements of the diagonal in the reproduced correlation matrix. They can each be written as

$$
\begin{aligned}
r^{* \prime}= & \left(h_{1}^{2}, r_{12}{ }^{*}, r_{13} *, \ldots, r_{1 N} *, h_{2}^{2}, r_{23}^{*}, \ldots,\right. \\
& \left.r_{i j}^{*}, \ldots, h_{N}{ }^{2}\right)
\end{aligned}
$$

or

$$
\begin{aligned}
r^{* \prime}= & \left(r_{12}{ }^{*}, r_{13} *, \ldots, r_{1 N^{*}}, r_{23} *, \ldots, r_{i j}^{*}, \ldots,\right. \\
& \left.r_{N-1}{ }^{*}\right),
\end{aligned}
$$

and

$$
\begin{aligned}
\hat{r}^{* \prime}= & \left(\hat{h}_{1}^{2}, \hat{r}_{12}{ }^{*}, \hat{r}_{13}{ }^{*}, \ldots, \hat{r}_{1 N}{ }^{*}, \hat{h}_{2}{ }^{2}, \hat{r}_{23}{ }^{*}, \ldots,\right. \\
& \left.\hat{r}_{i j}{ }^{*}, \ldots, \hat{h}_{N}{ }^{2}\right)
\end{aligned}
$$

or

$$
\begin{aligned}
\hat{r}^{* \prime}= & \left(\hat{r}_{12}{ }^{*}, \hat{r}_{13^{*}}{ }^{\prime}, \ldots, \hat{r}_{1 N} *, \hat{r}_{23} *, \ldots, \hat{r}_{i j}^{*}, \ldots,\right. \\
& \left.\hat{r}_{N-1 N}\right) .
\end{aligned}
$$

When the following form of linear regression is adopted after determining whether or not the elements of the diagonal in the upper (or lower) triangle matrices are to be included in the analysis

$$
r^{*}=\beta^{*} \hat{r}^{*}+e^{*},
$$

the value for $\beta^{*}$ is assumed to be unity when the assumed number of factors $m$ is to be close to the true one according to the formula (7). It seems natural that the answer for $\beta^{*}$ should be given as the value of $\beta$ in the formula (A-1) of Appendix based on the formula (A-3). However, it should herein be noted that the use of the letter $\beta^{*}$ rather than $\beta$ will 
be continued for further explanation as the both are same in their real meanings. Here $e^{*}$ denotes the column vector for the elements $e_{i j}{ }^{*} \mathrm{~s}$ in the matrix $R^{*}-$ $A^{*} A^{* \prime}$. If the assumption is correct, the value $\beta^{*}$ based on the result of the minres factor analysis may not change or may be unity without regard for the further increase of the number of factors after the reproduction of a correlation matrix has perfectly been achieved under an assumed number of factors.

\section{The Computational Procedure for the Minres Factor Analysis}

Let $r_{j}{ }^{+}(\mathcal{N} \times 1)$ and $r_{j}{ }^{0}(\mathcal{N} \times 1)$ be the $j$-th column of the correlation matrix $R^{+}$ each of the element of the diagonal of which is an initial estimated communality and the one of $R_{0}$, respectively. When the factor decomposition $R^{+}=A^{+} A^{+}$ under an assumed number of factors $m$ $(\leq \mathcal{N})$ is performed, $a_{j}{ }^{+}(m \times 1)$ denotes the $j$-th column of the matrix $A^{+\prime}$ which is the transpose of the matrix $A^{+}$. And let $A_{(j)}{ }^{+\prime}$ be the matrix $A^{+\prime}$ the $j$-th column of which is replaced by the vector $0(m \times 1)$ the elements of which are all zeroes.

The iterative refactoring method for the minres factor analysis can be performed through the minimization of

$$
Q=\sum_{j}^{N}\left(r_{j}{ }^{0}-A_{(j)}{ }^{+} a_{j}{ }^{+}\right)^{\prime}\left(r_{j}{ }^{0}-A_{(j)}{ }^{+} a_{j}{ }^{+}\right)
$$

with regard to $a_{j}^{+}$and the sum for (9) will be improved iteratively and will be minimized with regard to all the vectors $a_{j}{ }^{+} \mathrm{s}$. When the minimization and the process of the computation are achieved within the preassigned limit, the value of $Q$ is to be equal to the one for $2 \psi$ defined previously in the formula (7). Assuming the following linear relation

$$
r_{j}^{0}=A_{(j)}{ }^{+} a_{j}{ }^{+},
$$

Harman (1976) attained the estimated column vector $\hat{a}_{j}^{+}$

$$
\hat{a}_{j}{ }^{+}=\left(A_{(j)}{ }^{+\prime} A_{(j)}{ }^{+}\right)^{-1} A_{(j)}{ }^{+}{ }^{+}{ }_{j}{ }^{0}
$$

through the principle of least squares. As for the stabilization of the computational process, the value for the preassigned limit is assumed to be .005 in each of all the communalities according to the formula (7).

When the value of smaller than .005 , e.g., .001, is given for the one of the preassigned limit, more stable results may be attained. For example, with the increase of the number of factors, the values for $\beta^{*} \mathrm{~s}$ brought about through the iterations of the computation for the minres factor analyses may oscillate within smaller range than the ones with the preassigned limit of this time may do. However, the time for the computation will increase as the number of the variables in a given correlation matrix gets greater. Then, further investigation for the improvement of the computation for this type of factor analysis, which assumes the computational iterations of many times, should be needed for saving the time particularly from the practical point of view.

\section{Numerical Examples and Discussion}

Three kinds of correlation matrices are prepared to investigate the usefulness of the estimate which was discussed in this paper. The first kind of the correlation matrices are the hypothetical ones; Fruchter's 5 variables (1954), Harman's 6 hypothetical variables (1976), and, Thurstone's 26 box problem (1947). The second kind of them are the empirical ones; Chapman's 7 variables (1948), Harman's 8 physical variables (1976), Thomson's 8 variables (1951), Davis' 9 variables (1944), McCloy's 11 variables (1940), Harman's 16 variables rearranged by Shiba (1979) based on Harman's 24 variables, and, Harman's 24 variables (1976). Every number of factors for this kind of the correlation matrices was estimated subjectively by 
the analyst. And, the third kind of the correlation matrices are the ones which are constructed to examine some empirical aspects with regard to the factorial invariance from the statistical point of view. They are two cases for Bechtoldt's 17 variables I and II (1961).

The minres factor analyses both with the initial estimated communalities of unities and with the ones of SMCs are applied to the above correlation matrices, respectively. And the value for $\beta^{*}$ in the formula (8) is estimated under every number of factors for each matrix. In estimating the value for $\beta^{*}$, all the elements of the diagonal in the upper (or lower) triangle matrix in each of the original and the reproduced correlation matrices will herein be used. The obtained value might hopefully be more reliable and stable than the value based on the elements which do not contain the ones in the diagonal in each of the matrices, and our experiences indicate the hope.

The value of $\beta^{*}$ might be said to approximate almost monotonously to unity, but the monotonous trend may not be kept so smoothly. In other words, the values of $\beta^{*} \mathrm{~s}$ in the further increase of the number of factors may be said to oscillate within the range of rather smaller value after a great drop of the value for $\beta^{*}$ has once occurred in $a$ number of factors. The trend may be found in many numerical examples, e.g., the results through the minres factor analyses with the initial estimated communalities of unities in Fruchter's 5 variables, Harman's 6 hypothetical variables, Chapman's 7 variables, Harman's 8 political variables, McCloy's 11 variables, both cases of Bechtoldt's 17 variables, and, Harman's 24 variables.

The oscillation may be brought about through the iteration of the computation for the minres factor analysis, and it may occur rather often particularly in the correlation matrices of smaller number of variables. In addition to the phenomenon, different kinds of the initial estimated communalities may provide different answers in that kind of the matrices. For example, the differences between the result through the minres factor analyses with the initial estimated communalities of unities and the one with the initial estimated communalities of SMCs can be seen in the example of Harman's 6 hypothetical variables in Table 1. It is needless to say that, if the number of variables increases, the differences and the oscillation may tend to disappear. For example, see the both cases of Bechtoldt's 17 variables and Harman's 24 variables.

The true number of factors might be estimated through the investigation of the monotonous change of the values of $\beta^{*} \mathrm{~s}$ in the following manner. That is, if $\beta_{m} *$ and $\beta_{m+1} *$ denote the values based on the numbers of factors $m$ and $m+1$, respectively, and if the criterion concerning the absolute difference

$$
\left|\beta_{m} *-\beta_{m+1} *\right|<e\left(\beta^{*}\right)
$$

is defined, the smallest number of factors $m$ which attains the criterion (12) is assumed to be true. Taking account of the range of the oscillation brought about through the iterative computation for the minres factor analysis, it might be recommendable that the value for $e\left(\beta^{*}\right)$ is taken as .005 as we adopted the value for the preassigned limit in the computation for the minres factor analysis. The underline based on the criterion (12) suggests the true number of factors for each example in Table 1. Compared to the numbers of factors through the criteria concerning the number of factors; the weaker and stronger lower bounds of Guttman (shown as $S_{1}$ and $S_{2}$ in Table 1 , respectively), the assumed number of factors and the one estimated subjectively in the original study.

It may be better to keep in mind that the speed of the change of the values 
Table 1

Numerical examples

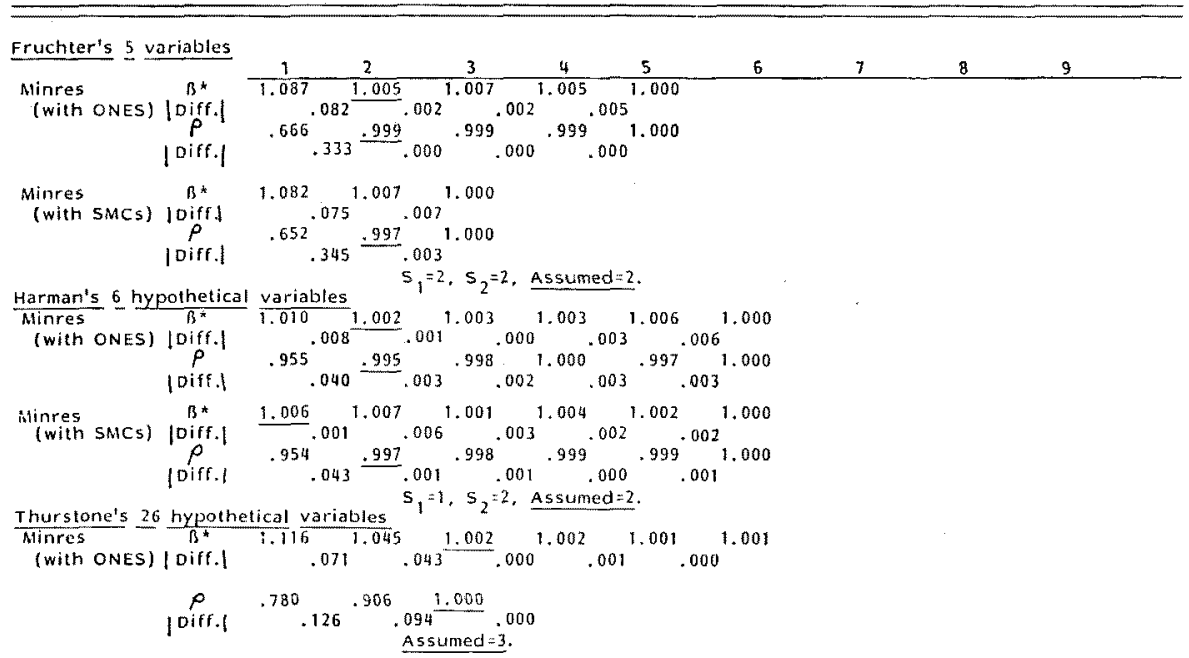

No solution by the minres factor analysis with the initial estimated communalities of SMCs can be obtained.

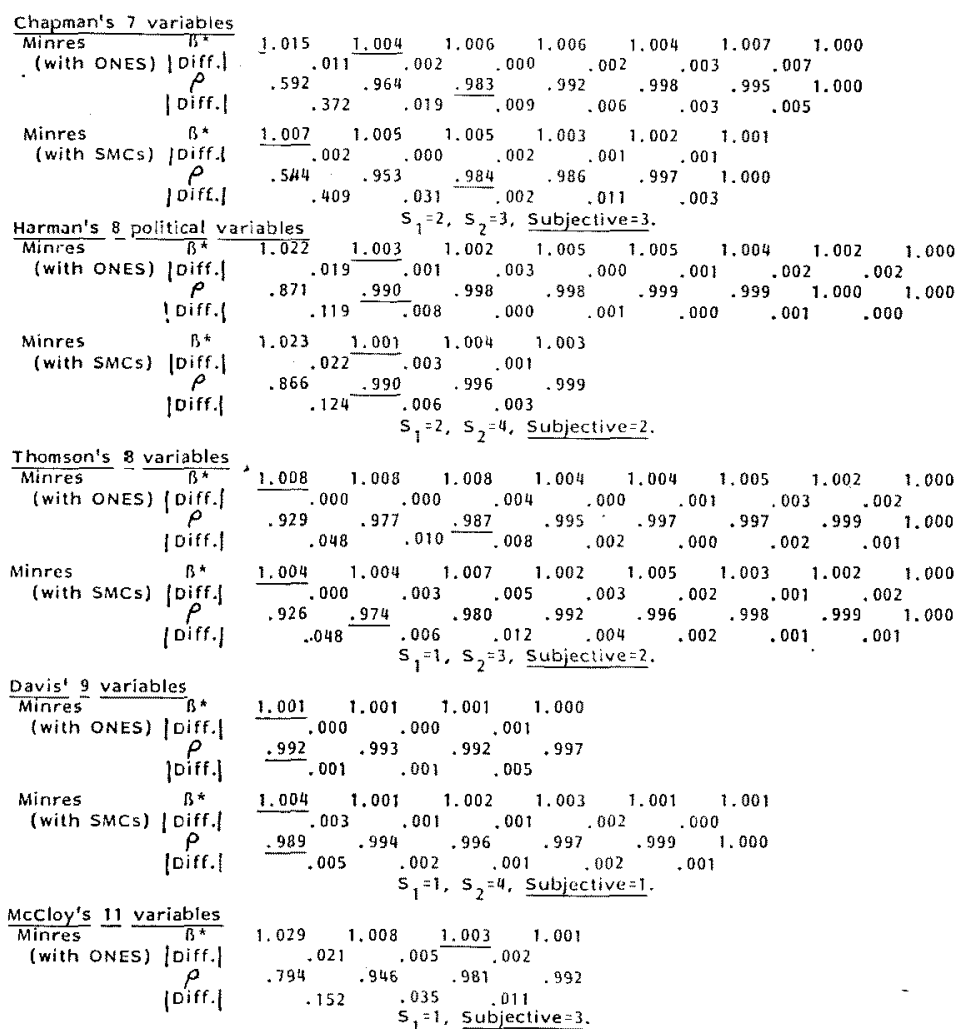

No solution by the minres factor analysis with the intial estimated communalities of SMCs can be obtained. 
Table I Continued

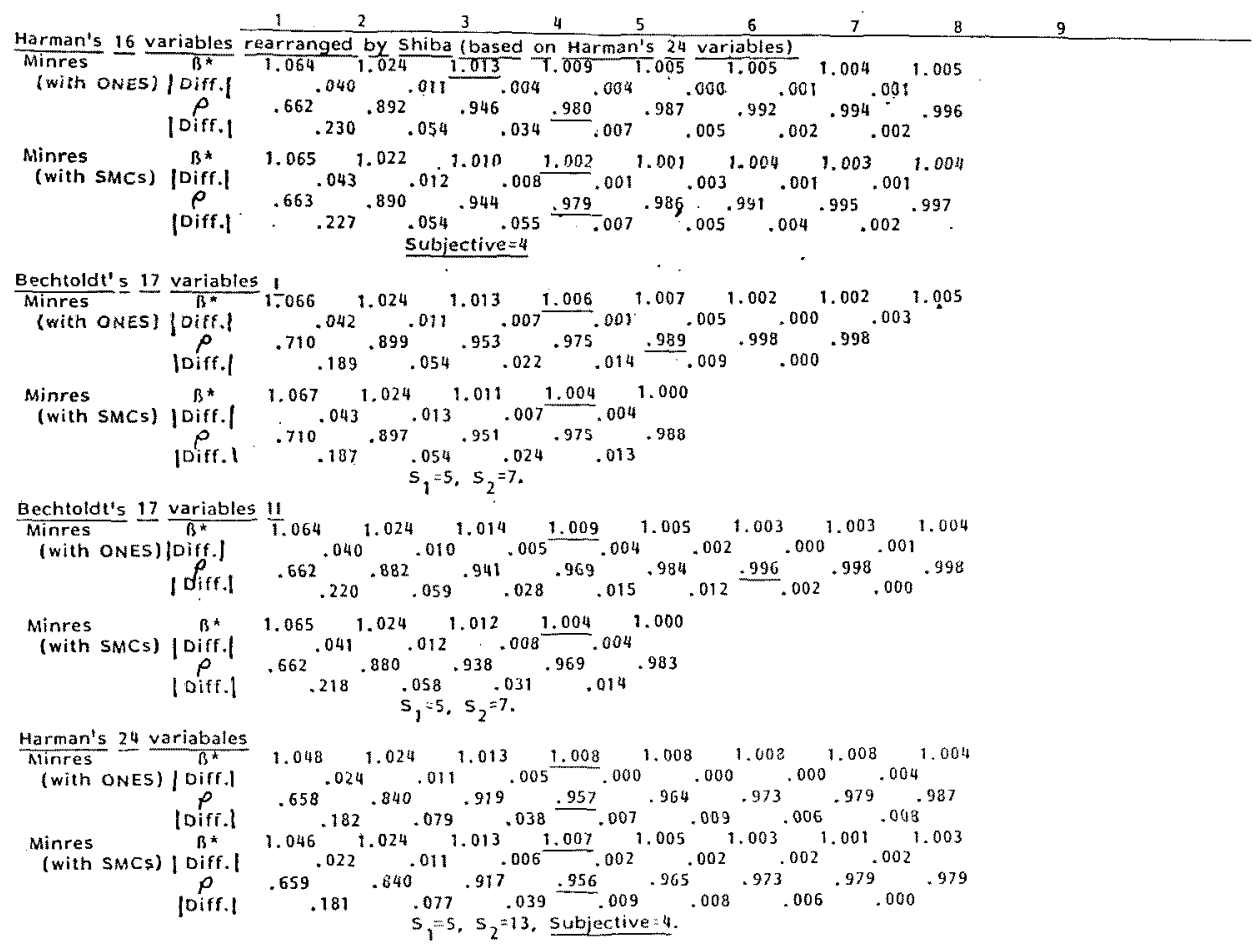

for $\beta^{*} \mathrm{~s}$ with the increase of the number of factors may happen to be slow so that they describe a longer or flatter tail. Then it may be harder to decide which number of factors should be adopted. If the case occurs, the following proposal based on Kashiwagi and Ishizuka (1973) from the practical point of view might be applied so as to secure the supplementary or additional information to the above criterion of (12) for the estimation of the number of factors.

When there is no negative element in its sign of the first factor brought about through the minres factor analysis, the coefficient of correlation $\rho$ based on all the elements in the upper (or lower) triangle matrix in each of the original and the reproduced correlation matrices can be calculated under a given number of factors. Here note that we include the elements of the diagonal of the upper (or lower) triangle matrix in each of the correlation matrices. The value for $\rho$ changes from -1 to 1 and hopefully it may not be greatly determined dependently upon the number of the variables in a given correlation matrix. The behavior of the values $\rho$ s with the increase of the number of factors might be suggestive for the estimation of the true number of factors. For example, if the increasing change of the values with the increase of the number of factors attains within less than a preassigned limit, the smallest number of factors $m$ which satisfies the limit in the succeeding one $m+1$ may be an estimate for the true number of factors. Following this kind of the idea, we could adopt the criterion

$$
\left|\rho_{m}-\rho_{m+1}\right|<e(\rho)
$$

where $\rho_{m}$ and $\rho_{m+1}$ denote the values brought about through the results based on the numbers of factors $m$ and $m+1$, respectively. It might be recommend- 
able that the value for $e(\rho)$ is taken as .01 , and it is assumed rather arbitrarily from the practical point of view. Of course, the assumption for this value should be examined more thoroughly in further studies.

The value for $\rho$ may be changeable when the variables in a given correlation matrix are reflected with regard to their signs. The possibility may occur when there is over one variable the sign of which is different from the rest ones in the matrix. Through their experiments, Kashiwagi and Ishizuka (1973) suggested that the reflections of the signs of the variables in a correlation matrix might bring about the different estimated value for $\rho$ compared to the estimated one for $\rho$ without assuming the reflections of the signs of the variables. Under an assumed number of factors, the estimated value for $\rho$ assuming the reflections of the signs of the variables may generally be larger. As this empirical fact seems to meet the idea concerning the factor parsimony of Thurstone (1947), the reflections of the signs of the variables in a correlation matrix which has over one negative element might be proposed before performing the minres factor analysis.

Then, if there is over one negative sign in the first factor brought about through the computation for the minres factor analysis, the signs of the corresponding rows and columns for both the original and the reproduced correlation matrices are to be reflected simultaneously under a given number of factors. And, the value for $\rho$ can then be calculated.

After estimating the value for $\rho$ under a given number of factors, the signs of the variables in the original correlation matrices should be unreflected. And then, the minres factor analysis will be continued under the succeeding number of factors.

The values $\rho$ s are presented in Table 1. The underline of the value in each example may indicate an estimate for the true number of factors. Compared to the numbers of factors brought about through the criteria; the weaker and stronger lower bounds of Guttman, the assumed number of factors, and, the number of factors estimated subjectively in the original study.

Before finishing the explanation in this paper, we should extend our consideration to the occurence of the Heywood case in the minres factor analysis. As Harman (1976) pointed out, only when a Heywood case happen to occur, such a type of the revised form for the minres factor analysis as proposed by him might be applicable. However, it should be noted that, only in order to obtain $\beta^{*}$, there seems to be no need to apply that type of the revision and it might be that the estimation of the number of factors is to be done irrespectively of whether or not the obtained result by the factor analysis is a Heywood case. One reason for the assertion comes from the author's impression, when the values $\beta^{*} \mathrm{~s}$ (some of which are assumed to be obtained through the revised form of the minres factor analysis) are obtained, almost entire monotonous change of the values $\beta^{*} \mathrm{~s}$ with the increase of the number of factors may happen to collapse rather greatly because of some partial applications of the revised form of the minres factor analysis under some numbers of factors.

\section{References}

Akaike, H. 1971 Determination of the number of factors by an extended maximum likelihood principle. Research Memorandum, No. 44, The Institute of Statistical Malhematics, March.

Bechtoldt, H. P. 1961 An empirical study of the factor analysis stability by hypothesis. Psychometrika, 26, 405-432.

Chapman, D. L. 1948 The MacQuarrie test for mechanical ability. Psychometrika, 13, 175-179.

Davis, F. B. 1944 Fundamental factors of comprehension in reading. Psychometrika, 9, 185197. 
Fruchter, B. 1954 Introduction to factor analysis. New York: Van Norstrand.

Guttman, L. 1953 Image theory for the structure of quantative variates. Psychometrika, 18, 277-296.

Guttman, L. 1954 Some necessary conditions for common factor analysis. Psychometrika, 19, 149-161.

Harman, H.H. 1976 Modern factor analysis. Chicago: University of Chicago Press.

Jöreskog, K. G. 1962 On the statistical treatment of residuals in factor analysis. Psychometrika, 27, 335-354.

Kaiser, H. F., \& Caffrey, J. 1965 Alpha factor analysis. Psychometrika, 30, 1-14.

Kashiwagi, S., \& Ishizuka, T. 1973 A linear regression criterion for the estimate of the number of factors. Japanese Journal of Psychology, 44, 167-178. (In Japanese with English summary)

Lawley, D. N., \& Maxwell, A. E. 1971 Factor analysis as a statistical method. 2nd ed. London: Butterwarths.

McCloy, C. H. 1940 The measurement of speed in motor performance. Psychometrika, 5, 173182.

Shiba, S. 1979 Inshi Bunseki Ho (Multiple factor analysis). University of Tokyo Press. (In Japanese)

Thomson, G.H. 1951 The factor analysis of human abilities. 5th ed. New York: Houghton Mifflin Co.

Thurstone, L. L. 1947 Multiple-factor analysis. Chicago: University of Chicago Press.

(Received Aug. 11, 1981; accepted Nov. 14, 1981)

\section{Appendix}

When the orthogonalized error $e_{i j}{ }^{*}$ in the formula (8) is expressed in terms of the non-orlhogonalized error $\varepsilon_{i j}$,

$$
e_{i j}^{*}=\varepsilon_{i j} \cos \theta=\left(r_{i j}^{*}-\beta f_{i j}^{*}\right) \cos \theta
$$

can be held. Of course, $\theta=\tan ^{-1} \beta$ where $\beta$ denotes the coefficient for the corresponding linear regression line by the principle of least squares based on the orthogonalized errors, $e_{i f}{ }^{*} \mathrm{~s}$.

Based on the relations described in the formula (A-1), the function

$$
F=\sum_{i<}^{N-1} \sum_{j}^{N} e_{i j} * 2=\sum_{i<}^{N-1} \sum_{j}^{N}\left(r_{i j} * \cos \theta-\hat{\vartheta}_{i j} * \sin \theta\right)^{2}
$$

is defined and the minimization with regard to $\theta$, i.e., $\partial F / \partial \theta=0$, is obtained. Then, the solution for this is to be

$$
\theta=1 / 2 \tan ^{-1} \frac{2 \sum_{i<}^{N-1} \sum_{i}^{N} \hat{f}_{i j}{ }^{*} r_{i j}{ }^{*}}{\sum_{i<}^{N-1} \sum_{j}^{N}\left(\hat{r}_{i j}^{* 2}-r_{i j}{ }^{* 2}\right)}
$$

From this,

$$
\beta=\tan \theta
$$

can be obtained. The above formula may give the beta coefficient $\beta^{*}$ shown in the formula (8) for the linear regression line to be estimated. 